\title{
QENS investigation of proton confined motions in hydrated perfluorinated sulfonic membranes and self-assembled surfactants
}

\author{
Quentin Berrod $^{1, \text { a }}$, Sandrine Lyonnard ${ }^{1}$, Armel Guillermo ${ }^{1}$, Jacques Ollivier ${ }^{2}$, Bernhard Frick ${ }^{2}$ and Gérard Gébel ${ }^{3}$ \\ ${ }^{1}$ Univ. Grenoble Alpes, CNRS/ CEA-INAC-SPrAM, 38000 Grenoble, France \\ ${ }^{2}$ Inst Max Von Laue Paul Langevin, 38002 Grenoble, France \\ ${ }^{3}$ Univ. Grenoble-Alpes, 38000 Grenoble, France; CEA, LITEN, DTNM, 38054 Grenoble, France
}

\begin{abstract}
We report on QuasiElastic Neutron Scattering (QENS) investigations of the dynamics of protons and water molecules confined in nanostructured perfluorinated sulfonic acid (PFSA) materials, namely a commercial Aquivion membrane and the perfluorooctane sulfonic acid (PFOS) surfactant. The former is used as electrolyte in low-temperature fuel cells, while the latter forms mesomorphous self-assembled phases in water. The dynamics was investigated as a function of the hydration level, in a wide time range by combining time-of-flight and backscattering incoherent QENS experiments. Analysis of the quasielastic broadening revealed for both systems the existence of localized translational diffusive motions, fast rotational motions and slow hopping of protons in the vicinity of the sulfonic charges. The characteristic times and diffusion coefficients have been found to exhibit a very similar behaviour in both membrane and surfactant structures. Our study provides a comprehensive picture of the proton motion mechanisms and the dynamics of confined water in model and real PFSA nanostructures.
\end{abstract}

\section{Introduction}

Proton exchange membrane fuel cells (PEMFC) are currently developed as clean and efficient alternatives to energy conversion technologies using fossil fuels. They are based on the use of nanostructured hydrated acidic polymers characterized by a hydrophilic/hydrophobic phase separation at the nanoscale. Perfluorinated sulfonic acid (PFSA) membranes such as Nafion ${ }^{\mathrm{TM}}$ from Dupont and Aquivion from Solvay (Fig. 1) are the actual benchmark ionomers, due to their good stability and high conductivity in hydrated states. However, they suffer from high cost, as well as stability limitations and insufficient conductivity at temperatures higher than $100^{\circ} \mathrm{C}$. Hence, the need for improving the membrane performances is an actual target. The PFSA proton conductivity is largely determined by the topology of the ionic phase and the proton-charges-water interactions. The morphology of the commercial membranes has been extensively studied in the last decades mostly by small-angle scattering techniques [1-4] and numerical simulations [5-9]. The swelling of percolating ionic nanodomains upon water loading (Fig. 1) is well established, however, their size, shape and connectivity are still discussed controversially. Various structural models have been proposed, including the organization of polymer ribbons into bundles [2], the hexagonal packing of water nanochannels [5] and the flat semi-lamellar topology [4]. As the structure is not unravelled, a microscopic understanding of the transportstructure interplay is still lacking. Proton transfer inside the electrolyte arises from a balance of i) hydronium ion diffusion, ii) peculiar motions at the polymer-aqueous

\footnotetext{
${ }^{a}$ Corresponding author: quentin. berrod@cea.fr
}

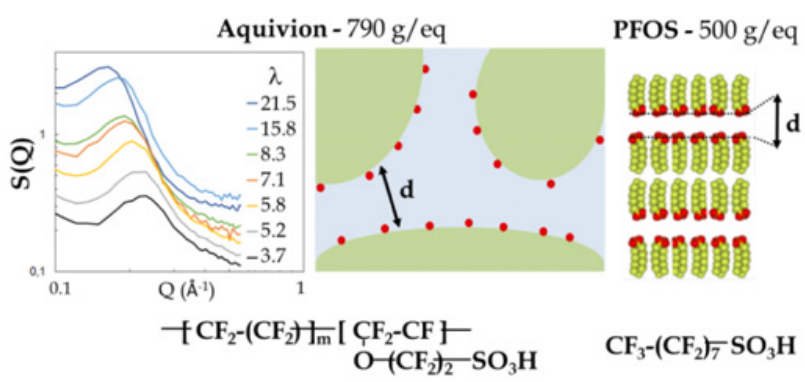

Figure 1. SANS spectra of Aquivion as a function of the hydration $\lambda$ from 3.7 to 21.5 , showing the shift of the ionomer peak position $\mathrm{Q}_{0}$. The characteristic d-spacing is obtained as $2 \pi / \mathrm{Q}_{0}-\mathrm{d}_{0}$. Chemical formula and schematic representation of the nanostructure of Aquivion membrane and PFOS surfactant.

phase interface, and iii) structural diffusion occurring by continuous breaking and forming of $\mathrm{H}$-bonds in the dense water molecules network [10].

The structural and dynamical properties of protons and water confined in the PFSA polymers have been extensively studied by numerical simulations [15-17] and infrared spectroscopy [18-23]. The short-side chain Aquivion was shown to perform better in fuel cells, with respect to the reference Nafion [11]. As both membranes possess similar nanostructures and comparable water selfdiffusion coefficients, the good performances of Aquivion were attributed to its higher density of charge, higher glass transition temperature and degree of crystallinity [12]. At a microscopic level, the confinement effect was shown to be more pronounced in Aquivion than in Nafion, but a better connectivity of ionic domains was evidenced. Hence, although confinement and connectivity effects are

This is an Open Access article distributed under the terms of the Creative Commons Attribution License 4.0, which permits unrestricted use, distribution, and reproduction in any medium, provided the original work is properly cited. 
differently balanced in Aquivion and Nafion, the overall impact of the polymer matrix morphology on the mobility of protons and water molecules turned out to be similar. Interestingly, only few Quasielastic Neutron Scattering studies were reported so far, none of them being dedicated to another polymer than Nafion. Yet, the QENS technique provides a unique insight into molecular dynamics, as a natural counterpart of the numerous numerical works.

In their pioneering QENS study, Volino et al. [24] analysed the motions of water molecules inside the Nafion polymer matrix with a model for the diffusion inside a sphere [25]. They showed the bulk-like behaviour of water in the fully hydrated polymer. Later, Pivovar et al. [26] used a phenomenological single-Lorentzian analysis based on Hall and Ross model [27] to quantify the observed acceleration of dynamics upon water loading. Finally, Perrin et al. analysed the behaviour of hydrated Nafion membranes from dry to fully saturated in an extended time range [28]. The hydration level was quantified by the local variable $\lambda$, defined as the number of water molecules per ionic group. It was shown that some protons, mostly belonging to hydronium ions, are localized in the vicinity of sulfonic acid groups and carry out very slow hops (typically hundreds of picoseconds). Diffusive motions appear for $\lambda>3$ and were analysed in the framework of the generalized Gaussian translational localized diffusion model [29]. It was shown that protons are confined inside water droplets (fast localized diffusion) and can diffuse from one droplet to a neighbouring one (nanometric longrange Fickian diffusion). Recently, we investigated the dynamics in perfluorooctane sulfonic acid (PFOS) (Fig. 1) used as a model system [30]. We showed that studying the motions of water and protons in the lamellar and hexagonal phases of these self-assembled structures could help to rationalize the more complex real membranes. Here, we report on a comprehensive study of the influence of the water degree of humidity ( $0 \%$ to $100 \%)$ on the local water and proton dynamics in both PFOS and Aquivion, i.e. a self-organized nanostructured model system and a stateof-the art short-side chain membrane. The QENS spectra over a wide energy range are obtained by combining time-of-flight (ToF) and backscattering (BS) experiments and analysed by a phenomenological multi-Lorentzian analysis approach based on microscopic models. This provides a detailed picture of the molecular mechanisms and delivers quantitative parameters such as characteristic times, confinement sizes, jump distances and diffusion coefficients.

\section{Experimental section}

\subsection{Materials}

The Aquivion membranes $(50 \mu \mathrm{m}$; equivalent weight $\mathrm{EW}=790 \mathrm{~g} / \mathrm{eq}$, i.e. $790 \mathrm{~g}$ of polymer per mole of sulfonic group) were purchased from Solvay Company. The asreceived membranes were acidified in nitric acid $(1 \mathrm{~mol} / \mathrm{L}$, $60{ }^{\circ} \mathrm{C}$ for 2 hours) and then rinsed in pure water ( 2 times at $60{ }^{\circ} \mathrm{C}$ for 2 hours) to insure full acidification. Strips of $3 \times$ $11 \mathrm{~cm}^{2}$ membranes were enclosed in cylindrical Al cells containing a reservoir filled with selected salt solutions. Various water vapor pressures were set to impose the
Table 1. Samples, RH and \%wt, $\lambda$ and confinement sizes $d$.

\begin{tabular}{|c|c|c||c|c|c|}
\hline $\begin{array}{c}\text { Aquivion } \\
\text { RH }(\%)\end{array}$ & $\lambda$ & $\begin{array}{c}\mathrm{d}(\AA) \\
\text { SANS }\end{array}$ & $\begin{array}{c}\text { PFOS } \\
\% \text { wt }\end{array}$ & $\lambda$ & $\begin{array}{c}\mathrm{d}(\AA) \\
\text { SAXS }\end{array}$ \\
\hline 11 & 3.7 & 3.3 & 95 & 1.5 & 3.7 \\
\hline 22 & 5.2 & 4.8 & 90 & 3.1 & 4.9 \\
\hline 52 & 5.8 & 5.9 & 85 & 4.9 & 7.1 \\
\hline 75 & 7.1 & 7.4 & 83 & 5.7 & 8.1 \\
\hline 85 & 8.3 & 9.1 & 75 & 9.3 & 9.5 \\
\hline 98 & 15.8 & 15.3 & 60 & 18.5 & 14.9 \\
\hline 100 & 21.5 & 19.9 & \multicolumn{4}{|l}{} \\
\hline
\end{tabular}

desired relative humidity $(\mathrm{RH})$, ranging from $11 \%$ to $100 \%$ (Table 1). From the sorption isotherm, we can convert a given RH into the water volume fraction $\Phi_{\mathrm{v} \text { water }}$ and the value of local hydration $\lambda$ (Table 1 ). The cells were rapidly pumped under vacuum, hermetically closed and then stored 3 days prior to QENS measurements to insure the $\mathrm{RH}$ equilibrium. The amount of strips located in each cell varied from $2(\mathrm{RH}>75 \%)$ to $8(\mathrm{RH}<30 \%)$ to maximize the counting rates without multiple scattering effects (transmission $>95 \%$ ). SANS measurements were conducted on the samples to evaluate the mean sizes of the ionic domains (Fig. 1), d, as a function of RH. The so-called ionomer peak is the fingerprint of the phaseseparation. $d$ is obtained from $2 \pi / Q_{0}-d_{0}$, where $Q_{0}$ is the ionomer peak position and $\mathrm{d}_{0}$ the mean size of hydrophobic aggregates obtained from the ionomer peak position extrapolated to zero water content.

The PFOS surfactant is commercially available (Sigma-aldrich) in aqueous solutions ( $\sim 40 \% \mathrm{wt}, \mathrm{EW}=$ $500 \mathrm{~g} / \mathrm{eq}$ ). The solutions were lyophilized. Surfactant mixtures were then prepared by adding water to obtain concentrations ranging from $\mathrm{c}_{\mathrm{s}}=40 \%$ to $97 \% \mathrm{wt}$. The PFOS phase-diagram obtained by SAXS shows a liquid (micelles)-to-gel transition at $\mathrm{c}_{s}=44 \%$ wt. Mesomorphous organized phases are formed upon surfactant concentration: hexagonal in the range [44-73\%wt] and lamellar at [83-97\%wt] [30]. The 2D SAXS spectra of PFOS samples were isotropic, as the lamellar or hexagonal oriented domains are randomly distributed in all directions. The mean sizes of the aqueous domains, d, are given in Table 1. For QENS experiments, PFOS gels spread in between 2 Teflon sheets ( $10 \mu \mathrm{m}$ thick) were enclosed in a $1 \mathrm{~mm}$ thick Aluminium flat container sealed with indium wire. The thin Teflon sheets were used to prevent degradation of the cell due to super acidity of the surfactants. As the sample transmissions were higher than $95 \%$, multiple scattering effects are negligible.

Table 1 summarizes the hydration state $(\lambda, \% \mathrm{wt}, \mathrm{RH})$ and the ionic domain sizes (d).

\subsection{Instruments and methods}

The ToF and BS incoherent QENS experiments were performed respectively on IN5 and IN16-B spectrometers at the Institut Laue-Langevin. Two incoming wavelengths of 8 and $5 \AA$ were used on IN5, yielding respectively an elastic resolution of $\Delta \mathrm{E}=20$ and $90 \mu \mathrm{eV}$ and Q-ranges of $\left[0.08-1.4 \AA^{-1}\right]$ and $\left[0.3-2.13 \AA^{-1}\right]$, where $\mathrm{Q}$ is the scattering vector. On IN16B the incoming 


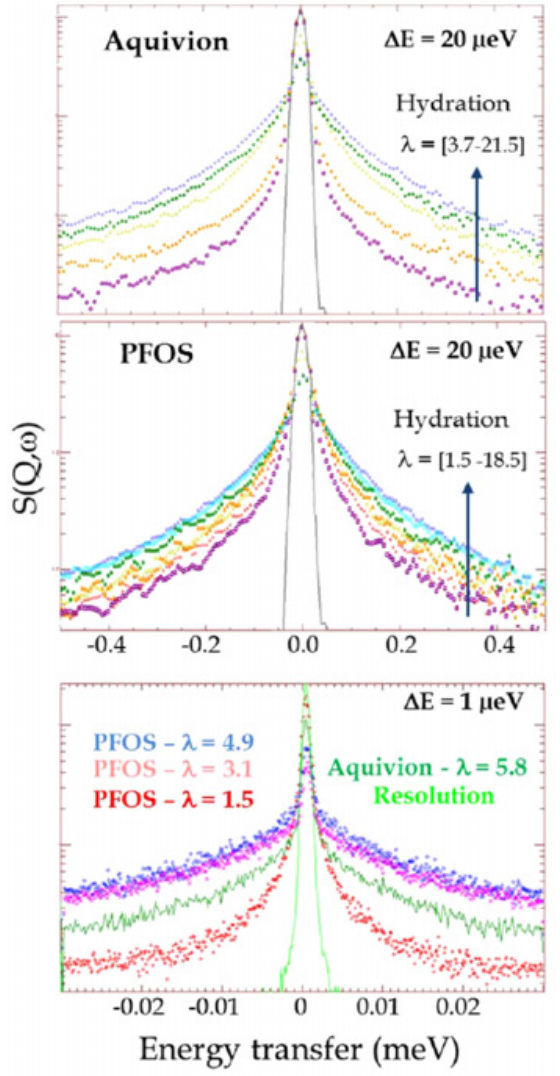

Figure 2. $\mathrm{S}(\mathrm{Q}, \omega)$ of Aquivion and PFOS at different hydration levels measured on IN5 $(\Delta \mathrm{E}=20 \mu \mathrm{eV})$ and IN16B $(\Delta \mathrm{E}=$ $1 \mu \mathrm{eV}$ ). The experimental resolution function is also shown.

wavelength of $6.271 \AA^{-1}$ yields an elastic resolution of $1 \mu \mathrm{eV}$ and a Q-range of [0.44-1.79 $\left.\AA^{-1}\right]$. The quasielastic spectra $\mathrm{S}(\mathrm{Q}, \omega)$, where $\omega$ is the energy transfer, were corrected from sample container and detector efficiency. A $0.5 \mathrm{~mm}$ thick flat piece of vanadium was used to obtain the experimental resolution function.

Some example spectra for hydrated Aquivion and PFOS are shown in Fig. 2. The quasielastic line is clearly evidenced at all investigated time-scales and is due to the incoherent scattering from hydrogen atoms. The coherent scattering due to the backbone is indeed at least 3 times lower than the incoherent one, and $\mathrm{ToF}$ measurements reveal no quasielastic signal in dry PFSA membranes [28]. The increasing broadening upon hydration shows the acceleration of motions in both PFOS and Aquivion with hydration level. Clearly, when comparing both samples we expect to find similar proton relaxation times as their Half-Width at Half Maximum (HWHM) are comparable. To fit simultaneously the QENS spectra for a hydration series measured on different spectrometers, we employed a multi-Lorentzian approach. The obtained HWHM and the integral of each component was then evaluated using different models (random jump diffusion model [27], Gaussian model [29]) to extract the diffusion coefficients, relaxation times, jump distances and confinement sizes.
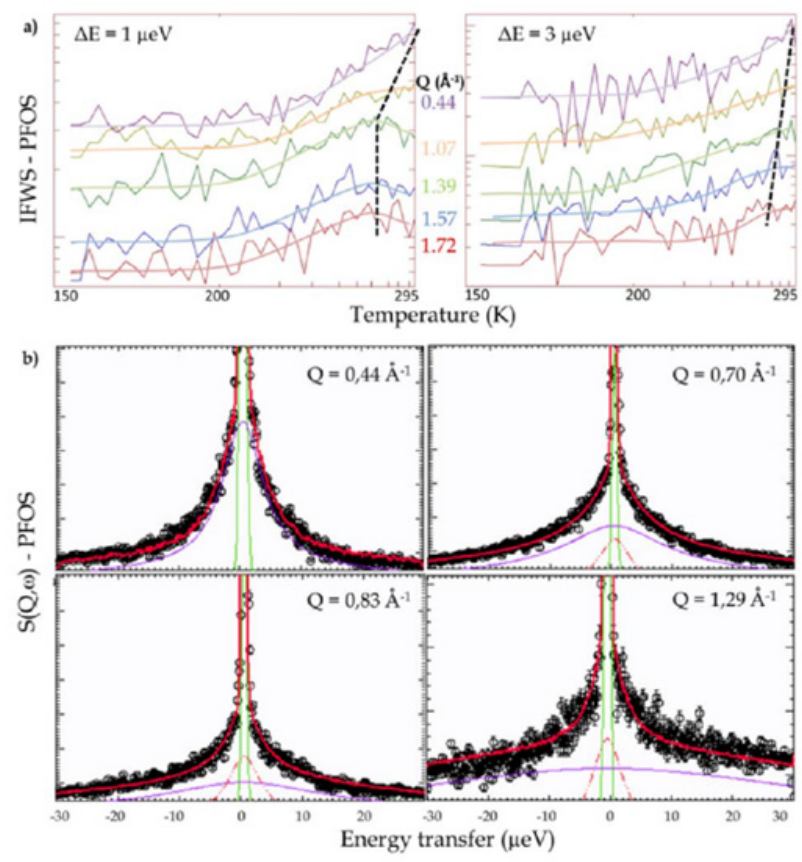

Figure 3. a) IFWS of PFOS at $\lambda=1.5$ b) IN16B $\mathrm{S}(\mathrm{Q}, \omega)$ spectra of PFOS $\lambda=3.1$ fitted with 2 Lorentzians. We observe a diffusive component (purple line) and a narrow one constant in Q corresponding to slow localized jumps (red dashed line).

\section{Results and discussions}

\subsection{Two populations of protons}

Previous studies on Nafion pointed out the existence of two distinct populations of protons on the basis of a careful analysis of high resolution BS spectra [28]. It was found that two Lorentzian components are required to adjust the quasielastic line over the whole Q-range. A narrow component with constant width (few $\mu \mathrm{eV}$ ) was found and attributed to the hopping of protons on a typical timescale of hundreds of ps and jump distances of $\sim 1 \AA$. The linewidth of the second broader component increases with Q and rapidly vanishes out of the BS spectral window, evidencing much faster diffusive motions, which are better resolved on the ToF spectrometer. We performed Inelastic Fixed Window Scans (IFWS) [31] on IN16B to evaluate the two-component hypothesis on the PFOS and Aquivion samples. The PFOS intensities obtained at two energy transfers, 1 and $3 \mu \mathrm{eV}$, are displayed in Fig. $3 \mathrm{a}$ in the temperature range [100-300 K].

At $1 \mu \mathrm{eV}$, we observe a temperature dispersive mode at low Q values (shift of an apparent peak to lower temperatures for higher Q [31]), evolving into a temperature-independent peak centered near $260 \mathrm{~K}$ for $\mathrm{Q}>1.07 \AA^{-1}$. In contrast, there is no indication of a peak at higher energy transfer $(3 \mu \mathrm{eV})$.

Although the maximum investigated temperatures are clearly too low, we can hypothesize that these data support the presence of a very narrow component characterized by a Q-independent width,that corresponds to slow localized motions (hundreds of ps). The dispersive mode observed at 
Table 2. Long range diffusion coefficient $\left(\mathrm{D}_{l r}\right)$, HWHM of the slow component with the corresponding jump time for Aquivion and PFOS, determined on IN16B.

\begin{tabular}{|l|l|l|l|}
\hline Sample & $\mathrm{D}_{\mathrm{lr}} \cdot 10^{5}\left(\mathrm{~cm}^{2} / \mathrm{s}\right)$ & $\Gamma_{\mathrm{S}}(\mathrm{meV})$ & $\tau_{\mathrm{S}}(\mathrm{ps})$ \\
\hline $\begin{array}{l}\text { PFOS } \\
\lambda=1.5\end{array}$ & $0.07 \pm 0.01$ & $3 \pm 0.5$ & $225 \pm 38$ \\
\hline $\begin{array}{l}\text { PFOS } \\
\lambda=3.1\end{array}$ & $0.27 \pm 0.02$ & $3 \pm 0.5$ & $225 \pm 38$ \\
\hline $\begin{array}{l}\text { PFOS } \\
\lambda=4.9\end{array}$ & $0.27 \pm 0.02$ & $3 \pm 0.5$ & $225 \pm 38$ \\
\hline $\begin{array}{l}\text { Aquivion } \\
\lambda=5.8\end{array}$ & $0.20 \pm 0.02$ & $2.5 \pm 0.3$ & $270 \pm 30$ \\
\hline
\end{tabular}

all energy transfer corresponds to faster diffusive motions. This is further confirmed by our attempts to adjust the quasielastic lines with a single Lorentzian, which failed. The data are well reproduced however by a two-Lorentzian fit as shown in Fig. 3b. The same results are found for the Aquivion membrane. The Q-dependence of the first narrow component (red dashed line) and the second broad one (violet line) is shown on Fig. 3b. The first component $(\mathrm{HWHM}=3 \mu \mathrm{eV})$ is undetectable at small Qvectors, increases in intensity but not in width with $\mathrm{Q}$, while the second one dominates at small Q, broadens significantly and then appears as a large background at high Q. It is noteworthy remarking that the new extended energy window of IN16B ( -30 to $30 \mu \mathrm{eV})$ allowed for an unambiguous discrimination of the two Lorentzians, in contrast to earlier measurements performed on IN16 ( -15 to $15 \mu \mathrm{eV}$ ) [28]. We can then analyse the Q-dependence of the HWHM and the corresponding intensities. A DQ ${ }^{2}$ behavior characteristic for long range (Fickian) diffusion is found for the broad component, with values of the selfdiffusion coefficients of PFOS and Aquivion membranes ranging from $0.07 \times 10^{-5} \mathrm{~cm}^{2} / \mathrm{s}$ to $0.27 \times 10^{-5} \mathrm{~cm}^{2} / \mathrm{s}$ (Table 2). The narrow component is due to a localized slow jump process of characteristic time $\tau_{s}=1 / \Gamma_{S}$. It thus appears that we have in these systems two types of protons, as was found in Nafion [28]. We can associate the slow hopping process to the restricted motion of protons strongly interacting with the sulfonate groups, thus mostly belonging to the hydronium species condensed at the hydrophobic/hydrophilic interface. In contrast, the number of fast diffusive protons is proportional to the water content, suggesting that these protons belong to water molecules.

\subsection{Fast diffusive motions}

The broad diffusive component evidenced on the high resolution BS data is further analyzed by inspecting the ToF data. First, we calculate the dynamic susceptibility function $\chi^{\prime \prime}(Q, \omega)$ defined as:

$$
\begin{aligned}
\chi^{\prime \prime}(Q, \omega) & \left.=S(Q, \omega) / \eta_{B}(\omega, T)\right) \\
\text { where } \quad \eta_{B}(\omega, T) & =\left(1-\exp \left(-\hbar \omega / k_{B} T\right)\right)^{-1}
\end{aligned}
$$

in order to identify the relaxation modes present in the various samples[36]. Very similar $\chi^{\prime \prime}(Q, \omega)$ are found in Aquivion and PFOS materials, with the presence of two

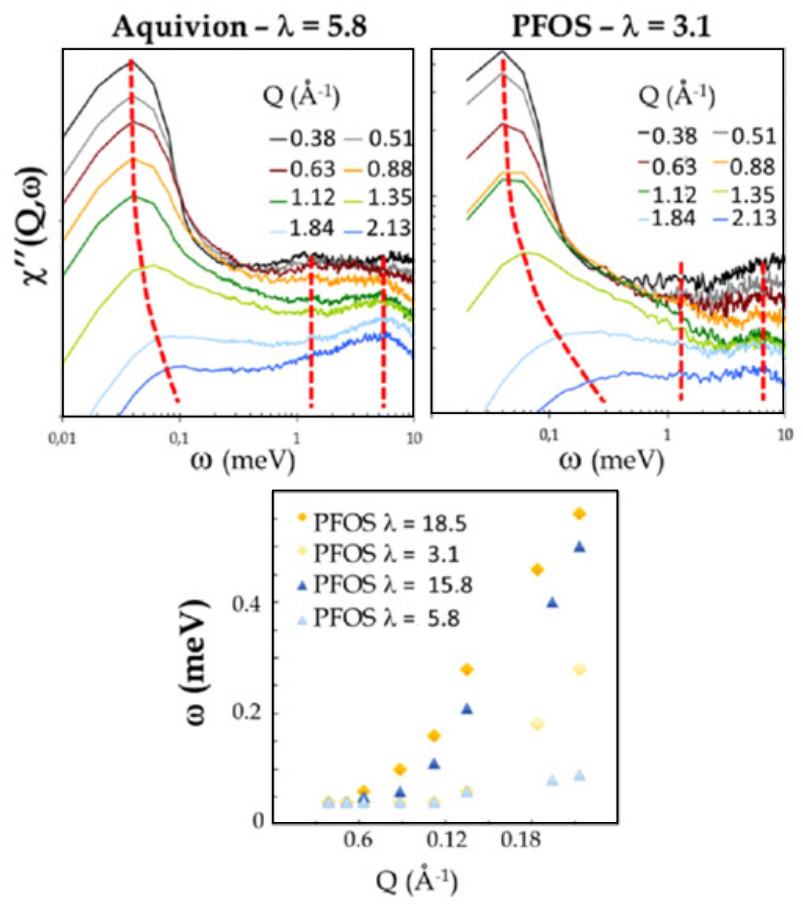

Figure 4. Susceptibility function $\chi^{\prime \prime}(Q, \omega)$ in Aquivion at $\lambda=$ 5.8 and PFOS at $\lambda=3.1$ plotted at different Q-values (top). The dispersion of the low-energy modes is increased with hydration (bottom).

Q-independent modes centered on 1 and $6 \mathrm{meV}$, and a low-energy mode appearing below $0.1 \mathrm{meV}$ (Fig. 4). The latter process is dispersive in $\mathrm{Q}$ and this effect is more pronounced when hydration increases (Fig. 4).

From the susceptibility representation it appears that $\mathrm{S}(\mathrm{Q}, \omega)$ spectra obtained on the ToF spectrometer arise from at least three different types of proton motion. Accordingly, the data cannot be reproduced by a single Lorentzian nor two Lorentzians. We fitted the spectra with the following function:

$$
\begin{array}{r}
S(Q, \omega)=D W\left[A_{1}(Q) L_{1}(\omega)+A_{2}(Q) L_{2}(\omega)\right. \\
\left.+A_{3}(Q) L_{3}(\omega)+P(Q)\right] \otimes \operatorname{Re}(Q, \omega)
\end{array}
$$

where $P(Q)$ accounts for an elastic contribution from the polymer matrix or surfactant chains, DW is the DebyeWaller factor and $\mathrm{L}_{i}$ are the three quasielastic contributions of HWHM $\Gamma_{i}$.

Figure 5a displays the various components of Eq. (2) and the global fit performed over an extended energy transfer region -3 to $0.2 \mathrm{meV}$. The PFOS and Aquivion spectra are consistently analysed at all water content, as highlighted on Fig. 5b at a given Q value. All data recorded at the two resolutions (20 and $90 \mu \mathrm{eV})$ are very well reproduced over the whole available Q-range, as seen from Fig. 5c.

The quasielastic widths $\Gamma_{1}, \Gamma_{2}, \Gamma_{3}$ extracted from the fits are plotted on Figs. 6 and 7 for the Aquivion and PFOS at all hydrations. $\Gamma_{2}$ and $\Gamma_{3}$ are found to be Qindependent, thus corresponding to the two fast localized 

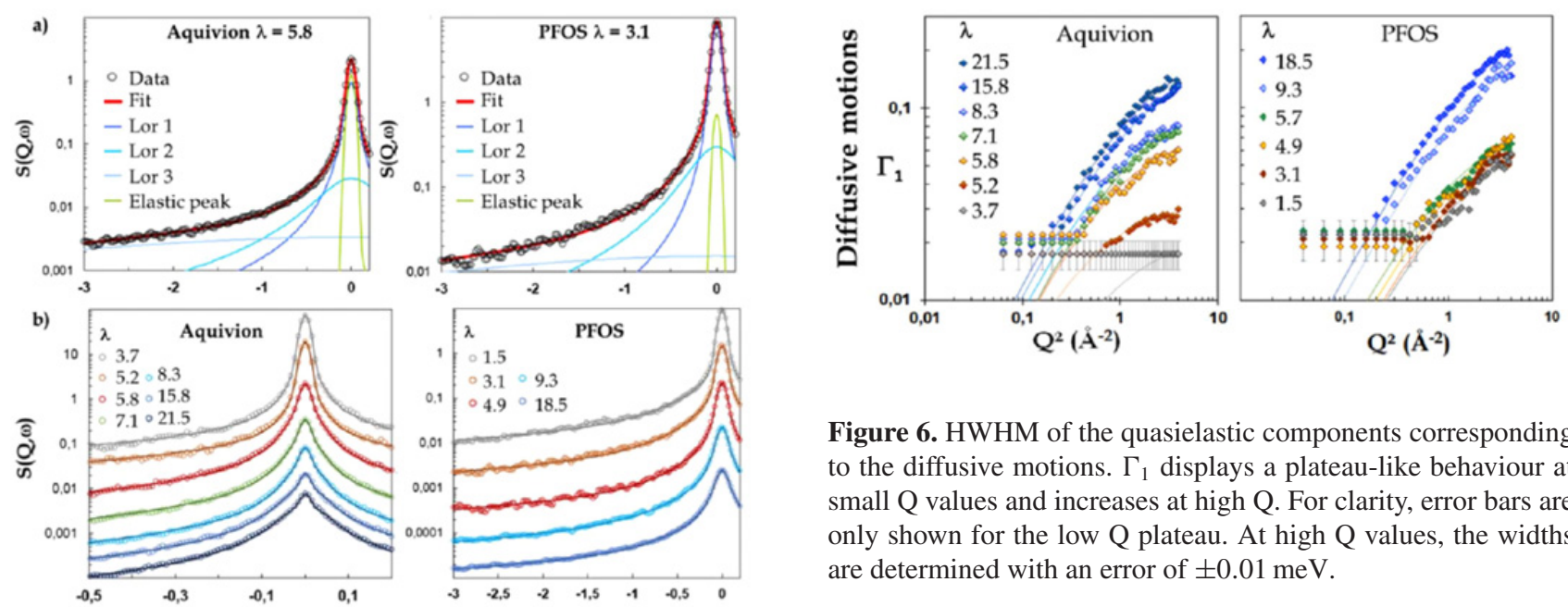

Figure 6. HWHM of the quasielastic components corresponding to the diffusive motions. $\Gamma_{1}$ displays a plateau-like behaviour at small $Q$ values and increases at high $Q$. For clarity, error bars are only shown for the low $\mathrm{Q}$ plateau. At high $\mathrm{Q}$ values, the widths are determined with an error of $\pm 0.01 \mathrm{meV}$.
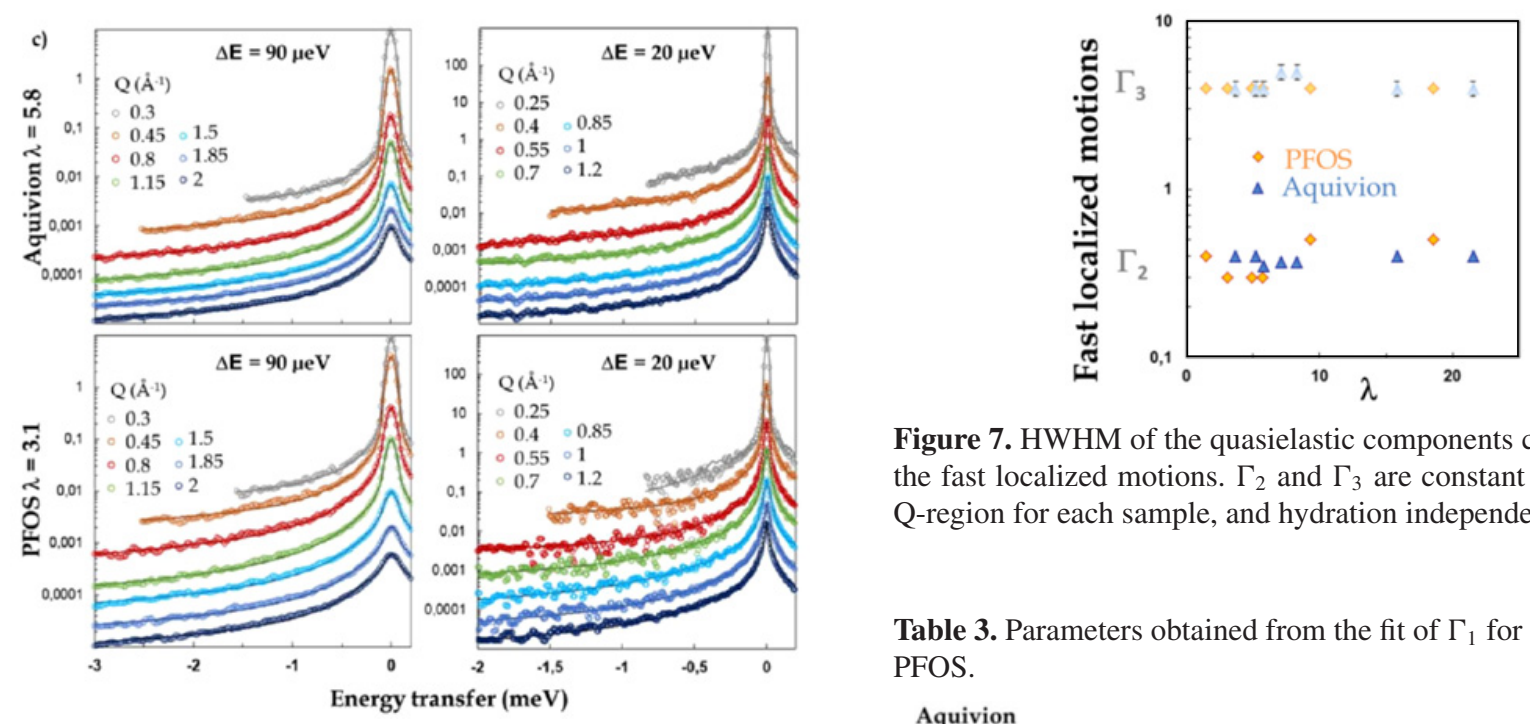

Figure 7. HWHM of the quasielastic components corresponding the fast localized motions. $\Gamma_{2}$ and $\Gamma_{3}$ are constant on the whole Q-region for each sample, and hydration independent.

Table 3. Parameters obtained from the fit of $\Gamma_{1}$ for Aquivion and PFOS.

\begin{tabular}{|c|c|c|c|c|c|c|c|}
\hline \multicolumn{8}{|c|}{ Aquivion } \\
\hline $\begin{array}{l}\text { HR } \\
(\%)\end{array}$ & $\lambda$ & $\begin{array}{l}2 \sigma \\
(\AA)\end{array}$ & $\begin{array}{c}D_{\text {H\&R }} \\
\left(10^{-5} \mathrm{~cm}^{2} / \mathrm{s}\right)\end{array}$ & $\begin{array}{c}\tau \\
\text { (ps) }\end{array}$ & $\begin{array}{c}u^{2} \\
\left(\AA^{2}\right)\end{array}$ & $\begin{array}{c}\Gamma_{2} \\
(\mathbf{m e V})\end{array}$ & $\begin{array}{c}\Gamma_{3} \\
(\mathbf{m e V})\end{array}$ \\
\hline 11 & 3.7 & $\cdot$ & $\cdot$ & $37 \pm 1$ & $0.3 \pm 0.1$ & $0.4 \pm 0.03$ & $4 \pm 0.4$ \\
\hline 11 & 3.7 & $2.5 \pm 0.3$ & $0.4 \pm 0.1$ & $30 \pm 2$ & $0.3 \pm 0.1$ & $0.4 \pm 0.03$ & $4 \pm 0.4$ \\
\hline 22 & 5.2 & $3.1 \pm 0.5$ & $1.0 \pm 0.1$ & $20.0 \pm 0.4$ & $0.3 \pm 0.1$ & $0.4 \pm 0.03$ & $4 \pm 0.4$ \\
\hline 52 & 5.8 & $3.7 \pm 0.2$ & $1.2 \pm 0.1$ & $8.6 \pm 0.6$ & $0.9 \pm 0.1$ & $0.35 \pm 0.02$ & $4 \pm 0.4$ \\
\hline 75 & 7.1 & $4.2 \pm 0.2$ & $1.20 \pm 0.05$ & $6.3 \pm 0.3$ & $1.2 \pm 0.1$ & $0.37 \pm 0.02$ & $5 \pm 0.5$ \\
\hline 85 & 8.3 & $4.4 \pm 0.1$ & $1.46 \pm 0.06$ & $6.4 \pm 0.2$ & $1.3 \pm 0.1$ & $0.37 \pm 0.02$ & $5 \pm 0.5$ \\
\hline 98 & 15.8 & $5.4 \pm 0.6$ & $1.7 \pm 0.1$ & $3.75 \pm 0.15$ & $1.4 \pm 0.1$ & $0.4 \pm 0.03$ & $4 \pm 0.4$ \\
\hline 100 & 21.5 & $5.6 \pm 0.4$ & $1.95 \pm 0.1$ & $3.3 \pm 0.1$ & $1.4 \pm 0.1$ & $0.4 \pm 0.03$ & $4 \pm 0.4$ \\
\hline \multicolumn{8}{|l|}{ PFOS } \\
\hline $\begin{array}{l}\% \mathrm{~m} \\
\text { PFOS }\end{array}$ & $\lambda$ & $\begin{array}{l}2 \sigma \\
(\mathbf{A})\end{array}$ & $\begin{array}{c}D_{\text {HSR }} \\
\left(10^{-5} \mathrm{~cm}^{2} / \mathrm{s}\right)\end{array}$ & $\begin{array}{c}\tau \\
(\mathbf{p s})\end{array}$ & $\begin{array}{c}<u^{2}> \\
\left(\AA^{2}\right)\end{array}$ & $\begin{array}{c}\Gamma_{2} \\
(\mathbf{m e V})\end{array}$ & $\begin{array}{c}\Gamma_{\mathbf{3}} \\
(\mathrm{meV})\end{array}$ \\
\hline 95 & 1.5 & $2.9 \pm 0.2$ & $0.68 \pm 0.10$ & $8.4 \pm 0.8$ & $0.9 \pm 0.1$ & $0.4 \pm 0.04$ & $4 \pm 0.4$ \\
\hline 90 & 3.1 & $2.9 \pm 0.2$ & $0.72 \pm 0.10$ & $7.6 \pm 0.7$ & $1.6 \pm 0.1$ & $0.3 \pm 0.03$ & $4 \pm 0.4$ \\
\hline 85 & 4.9 & $3.7 \pm 0.3$ & $0.85 \pm 0.10$ & $7 \pm 0.5$ & $1.4 \pm 0.1$ & $0.3 \pm 0.03$ & $4 \pm 0.4$ \\
\hline 83 & 5.7 & $3.6 \pm 0.2$ & $1.03 \pm 0.10$ & $7.5 \pm 0.6$ & $2.1 \pm 0.1$ & $0.3 \pm 0.03$ & $4 \pm 0.4$ \\
\hline 75 & 9.3 & $4.7 \pm 0.4$ & $1.64 \pm 0.05$ & $2.4 \pm 0.3$ & $1.0 \pm 0.1$ & $0.5 \pm 0.05$ & $4 \pm 0.4$ \\
\hline 60 & 18.5 & $5.8 \pm 0.8$ & $2.10 \pm 0.05$ & $2 \pm 0.2$ & $1.3 \pm 0.1$ & $0.5 \pm 0.05$ & $4 \pm 0.4$ \\
\hline
\end{tabular}

Figure 5. a) Examples of a three-Lorentzian fit of $S(Q, \omega)$ measured on the Aquivion at $\lambda=5.8$ and the PFOS at $\lambda=3.1$. Data are taken at $\Delta E=20 \mathrm{meV}$ and showed at $Q=0.8 \AA^{-1}$. b) Aquivion and PFOS spectra $\left(\Delta E=20 \mu \mathrm{eV}, \mathrm{Q}=0.8 \AA^{-1}\right)$ at all hydrations fitted with Eq. (2). c) Q-dependence in the two resolution configurations.

motions evidenced in the dynamic susceptibility function. There is almost no variations upon hydration and the values are found to be identical in PFOS and Aquivion, i.e. $\Gamma_{2} \sim 0.4 \mathrm{meV}$ and $\Gamma_{3} \sim 4 \mathrm{meV}$ (Fig. 7). The associated correlation times are equal to $\sim 1.6 \mathrm{ps}$ and $\sim 0.14 \mathrm{ps}$. Such motions are respectively compatible with the rotation of the water molecule ( $\tau=1.25 \mathrm{ps}$ in bulk water at room temperature) [32], and the life-time of Eigen or Zundel ions [33] or the H-bond vibration ( $100 \mathrm{fs})$ [34].

We will focus in this study on the first Lorentzian corresponding to the diffusive motions. Its width $\Gamma_{1}$ increases with $\mathrm{Q}$ and with hydration, with a plateau-like behavior typical of spatial confinement found at a $Q<Q^{*}$ (Fig. 6). The high-Q behavior can be analyzed by Hall and Ross model:

$$
\Gamma(Q)=D Q^{2} /\left(1+D Q^{2}\right) \tau
$$

with $\mathrm{D}$, the diffusion coefficient, and $\tau$, the elementary jump time. The quasielastic integral, $\mathrm{I}_{\mathrm{qel}}$, of component 1 , is well reproduced (Fig. 8) by the following model:

$$
I_{q e l}=D W \times A_{1}(Q)=D W \times\left(1-\exp \left(-Q^{2} \sigma^{2}\right)\right)
$$

where $\exp \left(-\mathrm{Q}^{2} \sigma^{2}\right)$ is the Elastic Incoherent Structure Factor (EISF) of a particle confined in a $2 \sigma$ size domain [29]. The polymer and surfactant elastic intensities exhibit a correlation peak around $\mathrm{Q}=1.2 \AA^{-1}$ due to the inter-chains correlations (Fig. 8) [3]. 

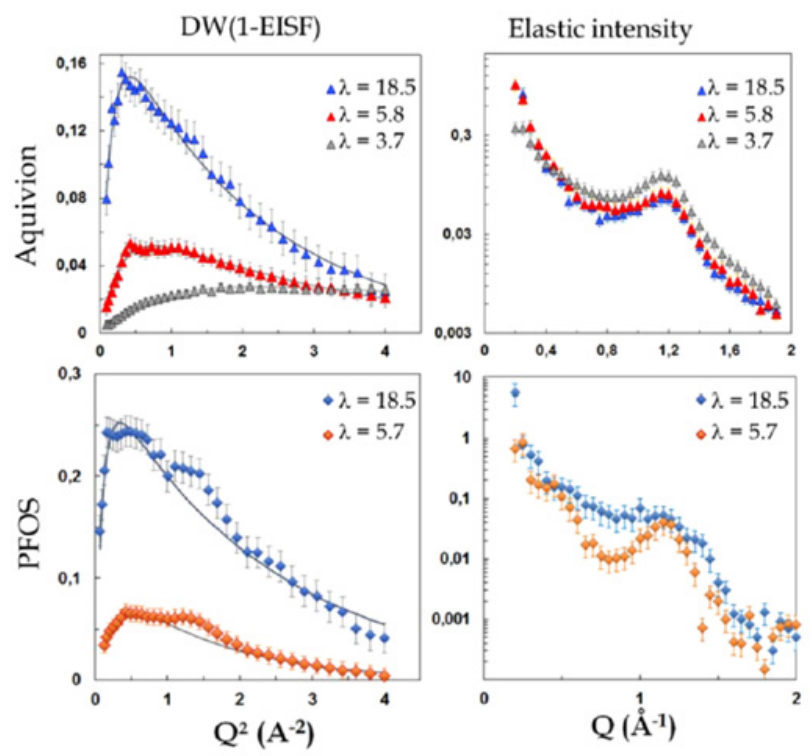

Figure 8. Fitted quasielastic integral and elastic intensities for representative Aquivion and PFOS hydrated samples.
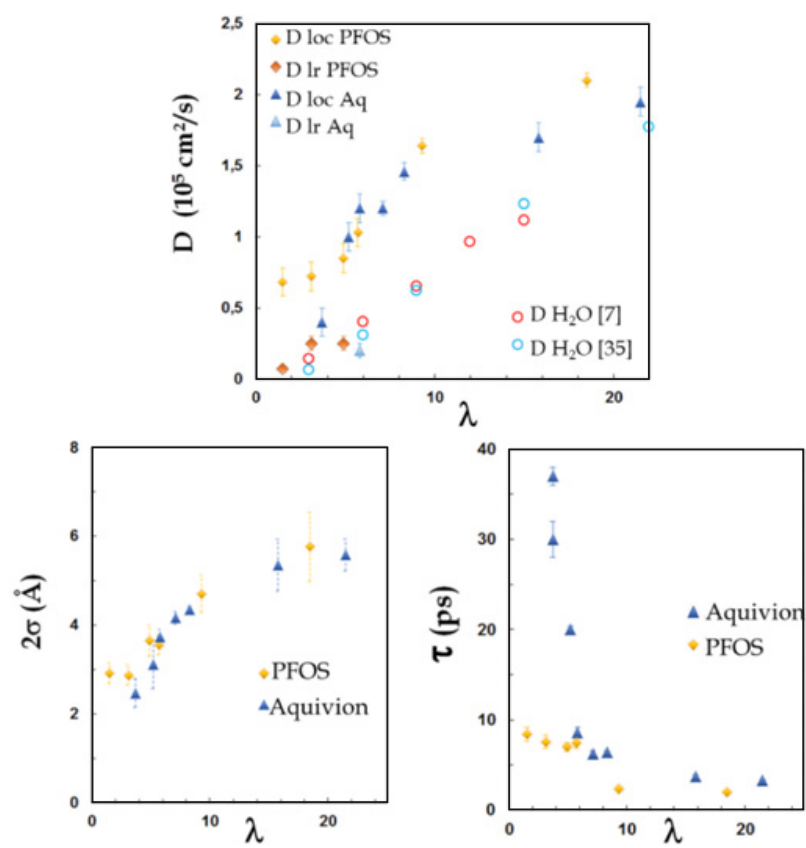

Figure 9. Diffusion coefficient D (top), elementary jump time $\tau$ and confinement size $2 \sigma$ (bottom). The diffusion coefficients computed from numerical simulations ([7] and [35]) are also reported.

\subsection{Molecular motions parameters}

The confined diffusive motions are characterized by the confinement size $2 \sigma$, the characteristic time $\tau$ and the local diffusion coefficient $\mathrm{D}_{\text {loc }}$. The variations of these parameters upon hydration in Aquivion and PFOS are plotted on Fig. 9 and compared to the long range diffusion coefficient $\left(\mathrm{D}_{\mathrm{lr}}\right)$ determined on IN16B. When the hydration is increased we notice i) the increase of the confinement size from $\sim 2$ to $4 \AA$, the increase of diffusion coefficient from 0.4 to $2.1 \times 10^{-5} \mathrm{~cm}^{2} / \mathrm{s}$, and iii) the associated diminution of the characteristic time until a minimum value of $2 \mathrm{ps}$. This behavior is the signature of an increased mobility of protons and water molecules towards an almost bulk-like behavior $\left(D_{\text {bulk }}=2.3 \times 10^{-5} \mathrm{~cm}^{2} / \mathrm{s}\right.$, $\tau_{\text {bulk }}=1.25 \mathrm{ps}$ ). It is remarkably similar in the polymer membrane and the surfactant molecules, although the nanostructure of the confining matrix is intrinsically different. The effect of confinement results in motions significantly slowed down below $\lambda<10$, as already observed in Nafion membranes[28]. At low hydrations $(\lambda<5)$ small differences appear between the two systems: i) D is slightly higher in PFOS ii) $\tau$ is smaller in PFOS and iii) $2 \sigma$ and d are smaller in Aquivion. Water dynamics is thus slightly faster in PFOS at low hydration and seems to reach a plateau, whereas the proton mobility is very sensitive to low hydration in Aquivion and decreases sharply. This saturation of the local dynamics in PFOS could result from its higher density of charge, favoring the surface diffusion from a sulfonate to another. The limiting factor would not be the confining geometry but the distances between two polar headgroups. It is noteworthy remarking also that long-range Fickian diffusion is reduced with respect to the local diffusion inside a confining water droplet of size $2 \sigma$, as seen on Fig. 9. Values of $\mathrm{D}_{\mathrm{lr}}$ are found to be similar in PFOS and Aquivion, and $\sim 3$ to 10 times lower than $D_{\text {loc }}$, as due to the nanoscale tortuosity effect. Interestingly, we can compare the values of the local and nanoscale diffusion coefficients to those extracted by Molecular Dynamics simulation performed on a short-side chain PFSA membrane [7] and Nafion pendant chain [35]. We notice that the water diffusion coefficients obtained by numerical approaches increase upon hydration, as expected. The values are found to be similar to the $\mathrm{D}_{\text {lr }}$ ones at low water content both in the membrane and in the surfactant, indicating that the localized translational motions of protons in confined water droplets is not accounted for in the MD simulations.

\section{Conclusions}

We have analysed quantitatively the dynamics of water molecules and protons confined in the Aquivion membrane and PFOS surfactant system, on an extended hydration range and time scale (from tenth of ps to hundreds of ps). A complex dynamical behaviour originating from nanoscale confinement in a charged nanostructured host matrix was found. Two populations of protons and similar processes were observed in both the state-ofthe art commercial membrane for fuel cells and the self-assembled PFOS structures. Slow localized proton jumps and long-range diffusion were identified from high resolution backscattering QENS data (on IN16B). Fast rotational motions and fast localized translational diffusion were obtained by ToF measurements performed at two different wavelengths. An accurate comparison of the effect of hydration on the hopping process, local and nanometric diffusive motions was obtained after a multi-Lorentzian analysis of the full set of QENS data. The results show that the local chemical environment (hydrophobic backbone with sulfonic acid terminations) plays a crucial role for the proton and 
water dynamics rather than the local organization of the nanostructure. Hence, self-assembled perfluorinated sulfonic acid surfactants appear to be relevant models for rationalizing the dynamical behaviour in more complex and disordered systems as polymer electrolyte membranes. This molecular-level study highlights the importance of local interactions on the mobility of ionic species in proton conducting materials.

\section{References}

[1] T. D. Gierke, G. E. Munn, and Fc. Wilson, J. Polym. Sci. Polym. Phys. Ed., 19, no. 11, pp. 1687-1704, 1981

[2] G. Gebel, Polymer (Guildf)., 41, no. 15, pp. 58295838, 2000

[3] L. Rubatat, A. L. Rollet, G. Gebel, and O. Diat, Macromolecules, 35, no. 10, pp. 4050-4055, 2002

[4] K. Kreuer and G. Portale, Adv. Funct. Mater., 23, no. 43, pp. 5390-5397, 2013

[5] K. Schmidt-Rohr and Q. Chen, Nat. Mater., 7, no. 1, pp. 75-83, 2008

[6] K. Malek, M. Eikerling, Q. Wang, Z. Liu, S. Otsuka, K. Akizuki, and M. Abe, J. Chem. Phys., 129, no. 20, p. 204702,2008

[7] J. Liu, N. Suraweera, D. J. Keffer, S. Cui, and S. J. Paddison, J. Phys. Chem. C, 114, no. 25, pp. 1127911292, 2010

[8] D. Wu, S. J. Paddison, and J. A. Elliott, Energy Environ. Sci., 1, no. 2, pp. 284-293, 2008

[9] R. Devanathan, A. Venkatnathan, and M. Dupuis, J. Phys. Chem. B, 111, no. 28, pp. 8069-8079, 2007

[10] K.-D. Kreuer, S. J. Paddison, E. Spohr, and M. Schuster, Chem. Rev., 104, no. 10, pp. 4637-4678, 2004

[11] A. Stassi, I. Gatto, E. Passalacqua, V. Antonucci, A.S. Arico, L. Merlo, C. Oldani, and E. Pagano, Journal of Power Sources, 196, No. 21, p. 8925-8930, 2011

[12] K. D. Kreuer, M. Schuster, B. Obliers, O. Diat, U. Traub, A. Fuchs, U. Klock, S. J. Paddison, and J. Maier, J. Power Sources, 178, no. 2, pp. 499-509, 2008

[13] J. Karo, A. Aabloo, J. O. Thomas, and D. Brandell, J. Phys. Chem. B, 114, no. 18, pp. 6056-6064, 2010

[14] D. Wu, S. J. Paddison, and J. A. Elliott, Macromolecules, 42, no. 9, pp. 3358-3367, 2009

[15] A. Venkatnathan, R. Devanathan, and M. Dupuis, J. Phys. Chem. B, 111, no. 25, pp. 7234-7244, 2007

[16] R. Devanathan, A. Venkatnathan, and M. Dupuis, J. Phys. Chem. B, 111, no. 45, pp. 13006-13013, 2007
[17] R. Devanathan, A. Venkatnathan, R. Rousseau, M. Dupuis, T. Frigato, W. Gu, and V. Helms, J. Phys. Chem. B, 114, no. 43, pp. 13681-13690, 2010

[18] E. Negro, M. Vittadello, K. Vezzù, S. J. Paddison, and V. Di Noto, Solid State Ionics, 252, pp. 84-92, 2013

[19] D. W. M. Hofmann, L. Kuleshova, B. D’Aguanno, V. Di Noto, E. Negro, F. Conti, and M. Vittadello, J. Phys. Chem. B, 113, no. 3, pp. 632-639, 2008

[20] D. B. Spry, A. Goun, K. Glusac, D. E. Moilanen, and M. D. Fayer, J. Am. Chem. Soc., 129, no. 26, pp. 8122-8130, 2007

[21] D. E. Moilanen, I. R. Piletic, and M. D. Fayer, J. Phys. Chem. C, 111, no. 25, pp. 8884-8891, 2007

[22] D. E. Moilanen, D. B. Spry, and M. D. Fayer, Langmuir, 24, no. 8, pp. 3690-3698, 2008

[23] R. Buzzoni, S. Bordiga, G. Ricchiardi, G. Spoto, and A. Zecchina, J. Phys. Chem., 99, no. 31, pp. 1193711951, 1995

[24] F. Volino, M. Pineri, A. J. Dianoux, and A. De Geyer, J. Polym. Sci. Polym. Phys. Ed., 20, no. 3, pp. 481496, 1982

[25] F. Volino and A. J. Dianoux, Mol. Phys., 41, no. 2, pp. 271-279, 1980

[26] A. M. Pivovar and B. S. Pivovar, J. Phys. Chem. B, 109, no. 2, pp. 785-793, 2005

[27] P. L. Hall and D. K. Ross, Mol. Phys., 42, no. 3, pp. 673-682, 1981

[28] J.-C. Perrin, S. Lyonnard, and F. Volino, J. Phys. Chem. C, 111, no. 8, pp. 3393-3404, 2007

[29] F. Volino, J. C. Perrin, and S. Lyonnard, J. Phys. Chem. B, 110, no. 23, pp. 11217-11223, 2006

[30] S. Lyonnard, Q. Berrod, B.-A. Brüning, G. Gebel, A. Guillermo, H. Ftouni, J. Ollivier, and B. Frick, Eur. Phys. J. Spec. Top., 189, no. 1, pp. 205-216, 2010

[31] B. Frick, J. Combet, and L. Van Eijck, Nucl. Instruments Methods Phys. Res. Sect. A Accel. Spectrometers, Detect. Assoc. Equip., 669, pp. 7-13, 2012

[32] J. Teixeira, M.-C. Bellissent-Funel, S.-H. Chen, and A.-J. Dianoux, Phys. Rev. A, 31, no. 3, p. 1913, 1985

[33] D. Marx, M. E. Tuckerman, J. Hutter, and M. Parrinello, Nature, 397, no. 6720, pp. 601-604, 1999

[34] J. Qvist, H. Schober, and B. Halle, J. Chem. Phys., 134, no. 14, p. 144508, 2011

[35] A. P. Sunda, and A. Venkatnathan, Journal of Material Chemistry A, 1, no. 3, p,557-569, 2013

[36] J.M. Zanotti, L.J. Smith, D.L. Price and M.L. Saboungi, J. Phys.: Condens. Matter, 18, p. 23912402, 2006 